Pascale GAULIN, «Yves Bonnefoy : la poésie au cœur du soupçon », @nalyses, printemps 2007

\title{
Pascale GAULIN
}

\section{Yves Bonnefoy : la poésie au cœur du soupçon}

En 140 pages, Olivier Himy s'est fixé comme but, dans son essai simplement intitulé Yves Bonnefoy, de faciliter l'accès à une œuvre complexe et riche, "d'en interroger les notions essentielles [...] de la rendre accessible à tous, du moins de donner à certains envie de la lire » (p. 6).

Le livre s'ouvre sur un " entretien imaginaire » avec le poète, qui «a été réellement soumis à Yves Bonnefoy, qui a bien voulu le valider comme tel » (p. 7). Ainsi l'interroge-t-il sur Breton et le surréalisme, le procès intenté au concept, "cette fatalité du langage» (p. 8), la notion de présence, l'image, les traductions et la question du langage. Si «le soupçon [est] porté envers le langage, [...] "c'est la fonction de la poésie de [le] réaccorder" » (p. 13). Mais, soutient Bonnefoy, «[o]n a bien droit de dire, en dépit de la critique des sémiologues, que la poésie peut prétendre à la vérité » (p. 13). Si la poésie "réaccorde » le langage, celui-ci permet à la poésie d'accéder au sens.

Les chapitres suivants s'articulent autour de trois grands axes: les "approches de l'homme», les "approches de l'œuvre» et les « approches thématiques».

Les éléments biographiques ainsi que le contexte littéraire et artistique qu'Himy met en perspective sont nécessairement factuels, mais donnent toutefois accès à l'origine et à l'évolution de l'œuvre de Bonnefoy. Si les événements, les rencontres, les dates et les titres marquent ce second chapitre, l'auteur y tisse de subtils liens qui prendront tout leur sens dans la section traitant de l'œuvre poétique et critique. Comme le précise Himy, "[] es éléments biographiques réunis montrent assez que l'œuvre comme la vie d'Yves Bonnefoy ne sont pas construites dans une superbe mais improbable solitude, mais au 
Pascale GAULIN, «Yves Bonnefoy : la poésie au cœur du soupçon », @nalyses, printemps 2007

contraire dans la proximité la plus grande avec les artistes et les intellectuels de son époque » (p. 21).

Le troisième chapitre propose une évolution des œuvres poétique et critique du poète, qui se dessinent en parallèle. Himy met d'abord l'accent sur l'origine et les métamorphoses de l'écriture de Bonnefoy, qui progresse de la prose vers la poésie. Les premiers textes sont indubitablement marqués par le surréalisme, bien que le poète s'en soit rapidement dissocié: "L'exploration surréaliste de l'inconscient permet ainsi au jeune poète de libérer une expression jusque-là enclose par le langage. À cet égard, l'écriture automatique apparaît comme un moyen, certes radical, de libération. Mais Bonnefoy en verra vite les pièges, et notamment l'impossibilité d'explorer plus avant les fantasmes par le biais de la conscience, comme celle de dépasser l'individu au profit de l'universel. » (p. 27-28) L'Anti-Platon et Le Courespace forment ainsi les premiers écrits du poète. La poésie de Bonnefoy restera par la suite marquée par une «tension [...] entre le réel et le rêve » (p. 39). Himy propose ensuite une brève analyse des Poèmes, rétrospective des quatre premiers recueils de Bonnefoy. Du mouvement et de l'immobilité de Douve, Hier régnant Désert, Pierre écrite et Dans le leurre du seuil sont liés par une progression thématique qui va du doute, de la présence de la mort dans la vie (Douve), à la lutte contre l'idéalisme et la perfection (Hier régnant désert), puis de l'ouverture à autrui, de l'acceptation de la vie, du rapport de l'écriture à la mort passant par une plus grande confiance en la poésie (Pierre écrite) et aboutissant à l'apogée d'une "réconciliation avec le monde » (p. 35) (Dans le leurre du senil). Il s'agit d'une œuvre traversée par l'existence, qui elle-même est la traversée d'une œuvre, qu'Himy dépeint avec justesse comme une quête initiatique. L'évolution de l'œuvre poétique de Bonnefoy repose sur la précision des liens qui en marquent l'unité formelle (prose/vers/récits en rêve) et sémantique, et ce, jusqu'à son plus récent recueil, Les Planches courbes. Cette unité s'étend également aux travaux critiques du poète. Himy précise l'ampleur de ce volet de l'œuvre en retenant «trois œuvres fondamentales dans la réflexion et l'évolution même d'Yves Bonnefoy, celles de Rimbaud, de Giacometti, et de Shakespeare » (p. 55), qui illustrent sa complémentarité critique : la critique littéraire, la critique d'art et la traduction. Poésie et critique 
Pascale GAUlin, «Yves Bonnefoy : la poésie au cœur du soupçon », @nalyses, printemps 2007

sont parallèles chez Bonnefoy, qui se dit à travers la critique, dont la poésie se nourrit. Une ambivalence ressort des écrits critiques de Bonnefoy, fortement marqués par le "soupçon contre le langage » (p. 58) qu'il porte et assume: "c'est bien le paradoxe, l'ambivalence, qui paraissent à Bonnefoy les fondements possibles d'une poétique » (p. 67). Himy suggère des pistes d'analyse et assume entièrement l'aspect lacunaire que peut présenter son approche.

Le quatrième et dernier chapitre porte sur les «approches thématiques", voire philosophiques de l'œuvre de Bonnefoy. Une analyse plus approfondie marque cette dernière partie de l'étude d'Himy. L'auteur y interroge notamment les notions de "présence», de «corps et figures », de «lieux », de «langage et [d']image», de «salut» que constitue la poésie même. L'auteur ayant déjà abordé quelques-unes de ces notions dans les chapitres précédents, cette section pourrait sembler redondante. Or, la finesse analytique oriente tout autrement les résultats. À des questions préalablement posées au fil des chapitres, Himy répond, apporte des conclusions. Ainsi, à la présence s'oppose le concept, " [p]arce que le concept apparaît à Bonnefoy comme une césure d'avec le monde» (p. 73). L'auteur met alors en relief l'ambiguité du fait que les écrits théoriques de Bonnefoy ont inévitablement recours au concept tout en le rejetant : «comment en serait-il autrement dès lors que sa réflexion prend bien souvent une tournure philosophique?» (p. 74). Mais « [1] a présence, on le comprend bien, n'est pas un concept » (p. 74). L'ambiguité est aussitôt résolue en considérant « que le rejet du concept et la quête de la présence sont le domaine de la poésie, et que le travail théorique n'en est qu'une explicitation, de laquelle la présence est nécessairement absente» (p. 74). La présence passe aussi par l'« immanence du simple» (p. 78), dont la poésie de Bonnefoy est très empreinte, tant par les éléments, les objets que par le lexique. Mais «[c]ertes, Yves Bonnefoy n’hésite pas à employer aussi des mots plus complexes, et surtout à faire usage de références mythologiques parfois rares » (p. 80). Ce ne sont là en fait que des «moyens en vue d'une fin, celle de saisir la présence, plus encore, celle d'essayer de la transmettre par la parole » (p. 80). À cela vient se joindre une «poétique du simple» (p. 83), qui se manifeste tant par la prose que par le vers, celui-ci «appara[issan]t alors comme la garantie de l'inscription du son dans la 
Pascale GaUlin, «Yves Bonnefoy : la poésie au cœur du soupçon », @nalyses, printemps 2007

parole, de l'inscription du corps dans l'écriture» (p. 84). La notion de " corps" prend dès lors une importance capitale en ce que celui-ci incarne le passage obligé de la souffrance menant à la présence: «Bonnefoy propose comme voie d'accès à la présence et à la saisie du monde un passage par la mort et la négation. [...] Parce que nous sommes des êtres de langage, nous sommes condamnés à ne saisir le monde que par ce biais imparfait des mots» (p. 90). Des figures féminines comme Douve et Cérès sont évoquées, habitant les poèmes, incarnant le « corps souffrant dont nous avons parlé, mais aussi le corps exultant» (p. 92). Ces figures «se tiennent "entre deux mondes", entre la vie et la mort, entre la souffrance et l'intransigeance, entre l'amour cherché et l'amour donné » (p. 96), et cet «entre-deux » est souvent représenté par le «fleuve » comme passage possible de l'un à l'autre. Le «passeur » y tient un rôle référentiel : «La figure du passeur serait alors liée à celle du sauveur.» (p. 99) Cependant, « [d]ans la perspective athée qui est celle d'Yves Bonnefoy, il ne faut pas y voir une tardive conversion, mais bien l'idée que le passage mène à une forme de salut, de salut du monde par les mots et la poésie, mais aussi de salut de la poésie par l'acceptation du monde et de la finitude» (p. 99-100). Moïse et Jésus représentent ces «sauveurs ", l'un d'abord sauvé des eaux, puis sauveur par la suite. L'enfant vient alors se substituer au sauveur, qui «sauve l'adulte» (p. 102). La notion de «lieu» prend ensuite toute sa place comme vérité recherchée: «La question posée par la notion de "vrai lieu", on le comprend aisément, n'est guère celle du lieu — la question où — que celle du vrai. [...] Aussi les lieux, réels ou non, n'importent chez Bonnefoy que dans la mesure où ils atteignent à un degré de vérité.»(p. 103, 107) Et ces lieux se situent tant dans l'« imaginaire » que dans « l'arrière-pays ». L'auteur consacre ensuite son propos à la question du langage, omniprésente dans l'œuvre de Bonnefoy. Au procès intenté au concept se substitue « une critique plus générale du langage même » (p. 110). Himy parle de « désécriture », «qui consiste à défaire dans le langage toutes les formes illusoires de la perfection» (p. 110). Par la critique du langage, l'image entre nécessairement en jeu, « tant est importante dans son œuvre la part prise par les images, aussi bien dans le travail critique que poétique » (p. 114). Critique du langage, de l'image; désécriture: il s'agit de procédés qui expriment le doute, le «soupçon» qu'Himy fait ressortir. C'est là 
Pascale GAULIN, «Yves Bonnefoy : la poésie au cœur du soupçon », @nalyses, printemps 2007

qu'émerge également toute la dimension métapoétique de la poésie de Bonnefoy. Ce travail sur le langage doit nécessairement aboutir à une «vérité de parole » qui transcende la matérialité du langage: "On voit alors que cette notion de vérité de parole, si chère à Bonnefoy, ne peut avoir d'autre expression que poétique, et qu'en tant que telle, elle restera sans doute toujours obscure à ceux qui n'entendent que le langage de la technique » (p. 121). Enfin, la poésie doit aboutir au salut, «la poésie est le salut » selon le propos de Himy (p. 122). Le salut passera par les mots, «malgré les mots » (p. 122), par la mémoire, la voix et la musique. Pour Bonnefoy, la poésie, incarnant tout cela, est le «moyen du salut» (p. 123).

Pour conclure son étude, l'auteur résume la poétique de Bonnefoy: «La parole poétique n'est pas un dépassement du langage, elle n'est pas non plus pure musique, mais elle permet la remémoration de l'intimité première avec le monde. » (p. 133) Bien qu'elle soit à la fois langage et émotion, la poésie devrait laisser apparaitre la «prééminence de l'émotion [...] sur le langage » (p. 128-129). Le salut de la poésie serait alors d'accéder au monde de l'enfant, de celui qui accède immédiatement au monde.

L'étude d'Olivier Himy sur Yves Bonnefoy est brillamment menée, compte tenu de la très grande densité de l'œuvre du poète. Son organisation, qui impose parfois des redites, conduit progressivement vers la poésie - vers la révélation de la poésie de Bonnefoy —, vers la réflexion sur la poésie, particulièrement présente dans le dernier chapitre. L'intérêt de cet essai réside surtout dans la présentation de l'auteur, car Himy ne pose pas de nouvelles questions sur l'œuvre. Si le mandat de ce livre était de rendre accessible l'œuvre de Bonnefoy, il n'est atteint que partiellement, car il faut assurément avoir d'abord lu et apprécié le poète et critique pour pouvoir ensuite mesurer les enjeux fondamentaux de son écriture.

Référence : Olivier Himy, Yves Bonnefoy, Paris, Ellipses, 2006, $143 \mathrm{p}$. 